ORIGINAL RESEARCH ARTICLE

\title{
Integrating Intimate Partner Violence Screening and Counseling in a Family Planning Clinic: Evaluation of a Pilot Project in Conakry, Guinea
}

\author{
Ghazaleh Samandari ${ }^{1}$, Alexandre Delamou ${ }^{2}$, Pernamou Traore ${ }^{3}$, Fatoumata Guilinty Diallo, \\ Sita Millimono ${ }^{5}$, Bienvenu Salim Camara ${ }^{6}$, Kira Laffe ${ }^{7}$, Fabio Verani $^{8}$ and Maimouna Tolliver $^{9}$
}

EngenderHealth, New York, USA ${ }^{1,5,7,8,9}$; Department of Public Health, Faculty of Medicine, University of Conakry, Guinea, Centre de formation et de recherche en santé rurale de Maferinyah, Guinea ${ }^{2}$; Association Guinéenne pour le Bien-Etre Familial, Conakry, Guinea $^{3}$; Ministry of Health, Conakry, Guinea ${ }^{4}$; Department of Public Health, Faculty of Medicine, University of Conakry, Guinea ${ }^{6}$

*For correspondence: E-mail: g.samandari@gmail.com; Phone: (212)561-8000

\begin{abstract}
Few programs exist to address Intimate Partner Violence (IPV) in Guinea. In 2014, Engender Health, in partnership with the local health authorities in Conakry, Guinea, piloted an integrated approach to IPV screening and counseling, within an existing family planning clinic. This article describes both the process of formulating and implementing this approach, as well as the results of an evaluation of the program. From January to June of 2014, Engender Health staff trained midwives at the Conakry International Planned Parenthood Federation family planning clinic staff in screening and counseling client for IPV. Program evaluators used project records, interview with program staff $(n=3)$, midwives $(n=3)$ and client exit interviews $(n=53)$ to measure the outcomes of this pilot project. Regardless of their IPV status, clients appreciated having a venue in which to discuss IPV. Program staff also felt empowered by the additional training and support for IPV screening. The evaluation yielded valuable suggestions for improvement, including more time for staff training and mock client interview practice, additional skills in counseling, and stronger referral links for women who screen positive for IPV. Integrating IPV screening into family planning services is an important and feasible method for reaching vulnerable women with IPV services. (Afr J Reprod Health 2016; 20[2]: 86-93).
\end{abstract}

Keywords: Sexual intercourse, sexual debut, risk factor, HIV/AIDS, IPV

\section{Résumé}

Peu de programmes existent pour répondre à la violence des partenaires intimes (VPI) en Guinée. En 2014, Engender Health, en partenariat avec les autorités sanitaires locales à Conakry, en Guinée, a piloté une approche intégrée au dépistage VPI et de conseil, dans une clinique de planification familiale existante. Cet article décrit à la fois le processus de formulation et de mise en œuvre de cette approche, ainsi que les résultats d'une évaluation du programme. De janvier à juin 2014, le personnel de EngenderHealth a formé des sages-femmes qui travaillent dans la clinique de planification familiale de l'International Planned Parenthood Federation dans le dépistage et le conseil du client pour la VPI. Les évaluateurs de programmes se servaient des dossiers du projet, des entrevues avec le personnel du programme $(n=3)$, sages-femmes $(n=3)$ et des entretiens de sortie des clients $(n=53)$ pour mesurer les résultats de ce projet pilote. Quel que soit leur statut de la VPI, les clients ont été contents d'avoir eu un lieu où ils pouvaient discuter la VPI. Le personnel du programme se sentait également habilité par la formation et un soutien supplémentaire pour le dépistage de la VPI. L'évaluation a donné de précieuses suggestions d'amélioration, y compris plus de temps pour la formation du personnel et de la pratique de l'entrevue de client fictif, des compétences supplémentaires en matière de conseil, et plus forts liens de référence pour les femmes dont le dépistage à l' 'egard de la VPI est positif. Intégrer le dépistage de la VPI dans les services de planification familiale est une méthode importante et réalisable pour atteindre les femmes vulnérables avec les services de la VPI. (Afr J Reprod Health 2016; 20[2]: 86-93).

Mots-clés: Les rapports sexuels, les premiers rapports sexuels, facteur de risque, le VIH / SIDA, VPI

\section{Introduction}

Intimate Partner Violence (IPV) is a human rights abuse and public health problem that affects women's physical, mental, sexual and reproductive health women in the world ${ }^{1,2}$. According to the World Health Organization (WHO) recent report, more than one in three women $(35.6 \%)$ globally experience physical and/or sexual violence, with highest rates in Sub- 
Saharan Africa ${ }^{3,4}$. The lifetime physical or sexual IPV prevalence or both vary from $15 \%$ to $71 \%$ in many countries ${ }^{3}$. Intimate partners perpetrate more than a third of female homicides ${ }^{5}$.

The risk of experiencing IPV is reported to be higher in adolescent and young women than older women ${ }^{6}$ and women victims of IPV are less likely to use reproductive health services ${ }^{7}$ and more likely to experience adverse reproductive health outcomes such as having low birth weight infants, pre-term delivery and neonatal death ${ }^{1}$. In Guinea, where the majority of the population is poor, rural and illiterate ${ }^{8}$, many women have experienced severe IPV, such as punching, kicking, threats or attacks with firearms or knives, strangling, forced sex, and unwanted sex acts ${ }^{9}$.

While some support for IPV survivors does exist in Guinea, there are still inroads to be made in providing comprehensive services for this population. One avenue for support is through integrating IPV screening into existing service delivery points, such as family planning clinics. This process not only allows survivors to select the most appropriate contraceptive method for their needs, but can also link them with additional services to deal with past or recurrent IPV ${ }^{10}$.

From January to June 2014, a pilot project for the integration of IPV screening and counseling into FP services was implemented in the adult family planning clinic of the Association Guineenne pour le Bien-etre familial (AGBEF), a member association of the International Planned Parenthood Association (IPPF) in Conakry, Guinea. The project conducted by Engender Health under the auspices of the USAID-funded RESPOND global program - was the first of its kind in Guinea, and was closely monitored to examine the process and outcomes of integrating IPV services into a family planning clinic. The objective of the Guinea IPV/FP Project, conducted in 2014, was to support improved reproductive health by integrating IPV screening and counseling into FP services in a low-income setting. Specifically, Engender Health pilot-tested a curriculum and supportive supervision model that trained AGBEF providers to meet a host of client needs, which included; 1) Screening for IPV with the FP client in a safe and supportive space; 2) Exploring FP method options with the IPV survivor; 3) Educating clients about medical, legal, psychosocial, and other services available to IPV survivors; 4) Discussing personal safety with the IPV survivor and helping her develop a safety plan for the future; and 5) Providing relevant medical services on-site or referral to other IPV services, as needed.

In this paper, we describe implementation research on integrating IPV screening into an existing family planning clinic; this study provides details on the operational processes of IPV/FP integration as well as results of an evaluation measuring the experience of providers, stakeholders and clients with the pilot program. In particular, we examine provider performance and satisfaction under the integration protocol, stakeholder analysis of feasibility of integrating IPV and FP services and client satisfaction with IPV screening as part of routine FP services. Findings from this pilot project can inform future scale-up or inception of IPV/FP integration projects in a variety of settings.

\section{Methods}

\section{Pilot program description}

The Guinea IPV/FP Project was a collaboration between Engender Health/RESPOND and local stakeholders in Conakry including local IPPF affiliate in Guinea, AGBEF. A steering committee responsible for monitoring project implementation was set-up by the Ministry of Health with members selected from the ministries of health, social affairs, security, and justice and from various partner organizations conducting IPV work in Guinea. The steering committee was responsible for participating in the planning of project activities, conducting field visits to monitor activities when needed, expanding the list of referral services, providing comments and suggestions on the IPV/FP service integration protocol and reviewing the project's progress and results of the intervention. An integration training protocol was developed by Engender Health using international guidelines with the purpose to inform the process of integrating IPV screening and counseling services into existing FP services. The protocol was reviewed and amended by the steering committee. 


\section{Training clinic providers in IPV screening}

The pilot project trained providers and other clinic staff $(n=4)$ in a 5-day training in February 2014, to improve their communication skills with clients and to foster a culture of confidentiality that protects the client's privacy with regards to IPV screening. The approach built on the GATHER model for FP counseling ${ }^{11}$ which the AGBEF clinic was already using, which combined the basic tenets of respectful and informed client interaction with guidance on where and how to integrate IPV screening and counseling by training staff to 1) provide clients with FP counseling based on the method of their choosing and; 2) conduct an in-depth method counseling and IPV screening after client's consent. Staff were trained to apply an IPV questionnaire to determine if the client was currently experiencing, or had experienced, psychological, sexual, or physical violence at the hands of a current or previous intimate partner (operational definition of IPV). Clinic staff were trained to provide those who screened positive with specific FP method counseling, taking into account the impact of IPV on method use, to develop a personalized safety plan and to refer the client to providers of IPV services in the community. Staff were also supervised closely by Engender Health staff and Ministry of Health trainers throughout the duration of the pilot.

\section{Study design}

We conducted a cross-sectional evaluation of the pilot project to determine the experience of providers, stakeholders and clients with the pilot project. In particular, we wanted to determine client satisfaction with IPV screening services, provider experiences with training and implementing IPV screening, and stakeholder opinions on the importance and feasibility of integrating IPV and FP services. Data were collected from March to June 2014, using qualitative and quantitative methods.

\section{Study site}

AGBEF is a Member Association of the International Planned Parenthood Federation (IPPF). The organization operates 9 clinics in
Guinea, including one youth clinic and one adult clinic in Conakry. The pilot IPV-FP integration approach was implemented at the adult family planning clinic in Conakry. The clinic staff included a nurse and midwife in charge of FP provision, antenatal care and sexually transmitted infections (STI) screening and care; a counselor who provided counseling and family planning methods to clients and support/administrative staff.

\section{Data collection}

We used project records, routine clinic service data, IPV screening tools, project meeting reports, key informant interviews to document the implementation of the project and to draw the lessons learnt. Service statistics and results from IPV screening from March to June 2014 were extracted from clinic records and analyzed to determine the rate and type of FP use and results of IPV screening among clients attending the clinic during the pilot period. Key informant interviews were held with stakeholders including members of the steering committee, clinic staff and managers and program staff and trainers $(n=12)$. Client exit interviews were used to determine women's experience and satisfaction with integrated services, which included a Likert score of their agreement with statements related to service provision (ranging from 1 indicating strongly disagree and 4 indicating strongly agree). All women receiving services for a 6 -week period during the pilot study were approached to be included in the interview. Of these, all 57 agreed to being interviewed but due to time constraints only 53 completed the full questionnaire and were included in the final analysis. Finally, pre-post training examinations were given to the 4 trained clinic staff to determine their comprehension of training material.

The evaluation plan was submitted to the Western Internal Review Board for ethical approval. All study participants were given informed consent before interview and no incentives were used.

\section{Analysis}

Quantitative clinic data were entered and analyzed in SPSS, using descriptive statistical 
methods. Likert scores were averaged across all women. Qualitative data were taped, transcribed and inductively analyzed using program objectives to identify salient themes. All analyses were reviewed and verified by a minimum of two Engender Health program staff.

\section{Results}

\section{Provider training and supervision in IPV/FP integration}

The pretest and posttest training examinations of clinic staff demonstrated improvements in several areas of their understanding and performance of integrated IPV screening services. As a result of the 5-day training, providers showed improvement in understanding of IPV and gender norms defining a safety plan and understanding the main elements of effective/active listening. This assessment, carried out directly by the training staff, also highlighted important areas requiring further strengthening, particularly the definition and process of patient confidentiality and the impact of provider attitudes and values on service delivery. The need for a close technical supervision during the first few weeks of implementation was also identified.

Based on the post-training provider feedback, an additional two-day session was organized to provide AGBEF staff with a review of the IPV screening process and to improve their mock client performance. The additional training resulted in vast improvements in mastery of IPV screening skills and improved provider attitudes towards IPV survivors.

Facilitative supervision was initiated at the AGBEF Clinic to ensure a clear understanding of the IPV screening and counseling process and a smooth flow of service provision for clients during the project implementation period. Due to the lessons learned during the initial and follow-on training, facilitative supervision visits were conducted twice a week for the first three months, and then once a week for the following three months of the pilot project to verify the institutionalization of procedures, record any challenges in capacity building and provide weekly project monitoring data. By the end of the pilot, more than 20 TA visits to the AGBEF clinic in Conakry had been conducted. The result of these visits demonstrated that although staff improved in their performance of IPV screening over time, they still struggled with the concept of confidentiality. Furthermore, they provided feedback to supervisors as to the additional burden of both time and emotional strain due to the length and intricacy of the IPV screening checklist. During TA visits, observers noted that the providers felt overwhelmed by the safety planning guide and would instead conduct oral safety planning with the client. This resulted in a lack of uniformity in the way in which services were being delivered.

\section{Provider experience with the IPV integration}

Providers felt that they were well-prepared to provide IPV screening and cited the importance of both the original and follow-on trainings, supervision, mock client interviews, and other practical experiences (e.g., filling out registration forms). Furthermore, they both mentioned that moral support helped them the most, followed by technical support/training and routine supervision.

"II felt I was adequately prepared to
provide IPV screening] because I
participated in the training, then
performed simulations during the
follow-on session that took place before
the start of clinic activities."--Nurse-
midwife

Screening was seen both as a means of providing an important service to women in the community and as an additional way for providers to meet clients' needs. The two providers said they would continue providing IPV screening and counseling services after the pilot.
"I know that women are interested, and I feel useful to my community. And I help women like me because we are all victims of violence."-Nurse-midwife
"Yes, we will continue to do the screening. Because it is very interesting, 
and women feel not only listened [to], but also protected."-Nurse-midwife

In addition to their positive feedback, providers offered suggestions for improvement of the integration process. Providers believed that the original training curriculum was too dense for a five-day period and should have included more practical skills building. They felt that the long safety plan (seven pages) was cumbersome for both themselves and clients and suggested shortening it to be more targeted to current IPV experience. Furthermore, they expressed a desire to have on-site counseling services to offer survivors and suggested building the clinic's capacity to provide psycho-social counseling as an immediate on-site benefit to women screening positive for IPV. Providers felt powerless to help women beyond the screening process, a burden which they described as traumatic to the provider.

\section{Women's IPV experience and changes in FP uptake}

IPV screening and counseling started at the Conakry AGBEF clinic on March 25, 2014. By the end of the pilot on June 2, 181 clients had visited the clinic, and 171 were screened for IPV (Table 1). The 10 clients who refused screening reported being in a rush to leave. Out of the 171 screened, 157 (91.8\%) screened positive for IPV (Table 1), meaning they reported experiencing one or more types of IPV. Three kinds of IPV were identified among them: sexual (68.2\%), psychological (79.6\%), and physical $(47.8 \%)$. The prevalence and correlates of IPV among participants have been published in a previous paper $^{13}$.

The nurse-midwife responsible for providing IPV screening and counseling offered safety planning to all clients who screened positive for IPV. A total of 137 clients completed safety planning (Table 1). The remaining women did not complete safety planning because of time constraints or a perceived lack of need. All women who screened positive for IPV received information about referral services, along with a list of contact numbers and addresses. However, the project documented only one case of a woman accepting referral.

Exit interviews with clients who received IPV screening and counseling were in May 2014. In total, 59 women (out of 59 approached) agreed to be interviewed, and 53 completed the entire interview. The women's mean age was 29 years, and $41 \%$ had secondary or higher level of education.

Women were asked to rate their experiences with IPV screening and counseling by agreeing/disagreeing with statements on a 4-point Likert scale (Table 2). Women strongly agreed that the provider explained things well (3.96), did not judge or blame the clients (4.0) and listened attentively (4.0). Women gave slightly lower marks on statements related to providers taking time to let clients ask questions (3.66) and feeling confident that providers would keep their discussion private (3.74).

When asked whether they thought IPV screening and counseling should take place during FP visits, all clients responded affirmatively. They noted the importance of raising awareness about violence among women and of providing a safe space for women to discuss this difficult topic.

"[IPV screening] is a good thing for women. We suffer, but we do not know what to do. Even if we suffer, parents say we must accept it so that our children can succeed tomorrow. What you started here is very good for us." Client, age 34, no education

All women interviewed said that they would recommend the clinic to other women and indicated that adding IPV screening did not dilute the quality of care provided.

"If the person is suffering like me, I have to tell her to come here for good advice." -Client, age 35, no education

\section{Stakeholder experience with IPV/FP integration}

Stakeholders on the steering committee and clinic management staff were interviewed and identified several successful aspects of the pilot project. 
Table 1: Number of IPV Screening and Counseling Activities among FP Clients at AGBEF Adult Clinic, Conakry, March-June 2014

\begin{tabular}{lllllll}
\hline $\begin{array}{l}\text { Frequencies (per month) } \\
\text { Activity }\end{array}$ & March & April & May & June & Total & $\%$ \\
\hline $\begin{array}{l}\text { No. of visiting } \\
\text { clients }\end{array}$ & 13 & 66 & 76 & 26 & $\mathbf{1 8 1}$ & \\
No. screened & 11 & 63 & 74 & 23 & $\mathbf{1 7 1}$ & $94.5 \%(171 / 181)$ \\
No. IPV-positive & 10 & 56 & 70 & 21 & $\mathbf{1 5 7}$ & $91.8 \%(157 / 171)$ \\
$\begin{array}{l}\text { No. who created a } \\
\text { safety plan }\end{array}$ & 0 & 44 & 70 & 23 & $\mathbf{1 3 7}$ & $87.3 \%(137 / 157)$ \\
No. referred & 0 & 0 & 1 & 0 & $\mathbf{1}$ & $0.6 \%(1 / 157)$ \\
\hline
\end{tabular}

Table 2: Mean Score of Clients on their Experience with IPV Screening and Counseling, AGBEF Adult Clinic, Conakry, May 2014

\begin{tabular}{ll}
\hline Statement & Score (out of 4) \\
\hline The provider explained things in a manner that I could understand. & 3.96 \\
The provider took the time to let me ask questions. & 3.66 \\
The provider told me that our conversation would be confidential. & 3.83 \\
The provider listened attentively when I had something to say. & 4.00 \\
The provider did not judge or blame me. & 4.00 \\
I believe the information I received is accurate. & 3.98 \\
I am confident that the provider will keep our discussion private. & 3.74 \\
\hline
\end{tabular}

Note: Scores could range from 1 (strongly disagree) to 4 (strongly agree).

Respondents felt that the training and close supervision were effective in building local capacity to screen for IPV and that it was simple enough to adapt and replicate in other settings. The data management tools were also singled out as effective means of collecting important client data for program use.

\section{"Despite the short duration of the project, our conclusion is that the project has not only affected a number of women, but it has strengthened the technical skills of providers and supervisors involved in the project." - Steering committee member}

Beyond having programmatic benefits, the pilot was viewed as an essential service to women in the community. Stakeholders acknowledged the need to integrate IPV screening into FP services in Conakry and felt that future scale-up of the approach could benefit women across Guinea.

"We realized that the need [for IPV screening] is there and it is real; the project is consistent with the needs of the population. Scale-up is necessary to benefit more women." - Steering committee member

One steering committee member reported that the pilot had built institutional capacity within the IPPF network, which could be leveraged to propagate IPV screening skills in other IPPF member clinics:

"The project has strengthened the institutional capacity of our [member association]. We want to develop the same skills at other clinics." - Steering committee member representing an IPPF member association

Stakeholders felt that providers need monetary compensation for the added workload that IPV screening and counseling entails, particularly as a motivation to continue the work. Because of the limited uptake of referral services during the pilot, respondents recommended that psychosocial counseling be available on-site, and that perhaps training could be provided for clinic staff to provide psychosocial support. Finally, one respondent suggested including a community outreach component that would reach women beyond the clinic with information about IPV and related services. 


\section{Discussion}

This pilot project to integrate IPV screening and counseling into FP services in a low-income setting demonstrated that the approach is viable. When providers are well-trained and receive adequate training and supervision, they can gain the skills needed to offer essential IPV screening and counseling services. From the clients' perspective, the integration of IPV screening and counseling into FP services is both needed and appreciated, as it allows women to discuss an issue that is culturally taboo in many settings. Including IPV screening did not negatively impact clients' willingness to use existing FP services. On the contrary, most clients accepted and appreciated the opportunity to speak out about their suffering and explore ways of coping with the impact of violence and develop measures to increase their levels of safety through safety planning. Although this project was the first of its kind in Guinea, other studies have demonstrated the utility of adding IPV screening into existing services where at-risk women may be reached, including HIV clinics ${ }^{14}$ and primary care services ${ }^{15}$.

The use of the curriculum and post-training follow-up revealed several important lessons about how to create a successful IPV-FP integration curriculum. First the curriculum should be highly practical and adapted to the educational and skill levels of trainees. It should include more time spent on operational aspects of IPV-FP integration such as mock client interviews and practice using reporting forms. Participants in training sessions also need more time to absorb, review, and practice the curriculum content. While the curriculum is important, other essential capacitybuilding elements include on-the-job training, mock-client visits, practice filling out reporting forms, and supervision. Both providers and trainers in the pilot felt that these additional elements were successful and essential to operationalizing learning.

Providers were responsible for all IPV screening and counseling, as well as their regular FP duties. This led not only to an increased burden of duty for providers, but also the experience of secondary trauma, resulting from the exposure to clients' IPV stories. Studies have shown that providers who care for women in traumatic situations do experience emotional fatigue and secondary trauma, which can have real consequences for the providers' well-being and performance ${ }^{16,17}$. To spread out the burden and to encourage sharing, clinics should have more than one provider conducting IPV screening and counseling. Clinic management should ensure adequate emotional support to prevent or manage secondary trauma among providers screening for IPV.

Throughout the project, clients who screened positive for IPV received a list of referral agencies available in the community. However, very few women expressed an interest in visiting referral sites; the project is aware of only one client who did so during the project. This situation was demoralizing to the provider, who viewed the lack of referral uptake as a mark on her performance, when, in fact, this is quite common behavior among survivors of intimate partner violence $^{18}$. This issue raises a larger question as to the ethics and utility of interventions focused only or primarily on screening. The providers we trained themselves expressed a desire to have psychosocial services available on-hand at the clinic to deal with IPV survivors as they are screened. Scholars of IPV interventions have also argued the importance of going beyond screening, with strong linkages to psychosocial services ${ }^{19}$.

Due to project time and budget constraints, staff were unable to follow-up with clients to investigate reasons for the lack of referral uptake. Although refusal to engage with referral services is common among IPV survivors, project staff and providers together reflected on the potential contextual reasons why clients were reluctant to use referral services. The sociocultural context, which is characterized by normalization of IPV and a taboo about discussing IPV, may stigmatize visiting services that are aimed specifically at IPV survivors. Furthermore, describing personal experiences of IPV can be difficult or traumatic for survivors; the prospect of repeating those experiences at a second location may be a barrier to referral uptake. The additional cost in money and time may also be prohibitively burdensome for some clients, particularly women who are responsible for the care of multiple children. 


\section{Conclusions}

The integration of IPV screening and counseling into FP services is a viable and acceptable approach both for providers and for clients. The findings from this pilot project can be used to sustain and scale up the approach in Guinea and to adapt it for other, similar contexts. Even though the project demonstrated successes, this type of programming would work best as part of a broader multi-sectoral IPV initiative, with IPV screening and counseling in FP clinics as only one element of the larger approach.

\section{Contribution of Authors}

Drs. Delamou and Samandari conceived of and designed the study, collected and analyzed the data, and prepared the manuscript. Drs. Millimono, Diallo, Traore and Camara contributed to program implementation, data collection and manuscript review. Kira Laffe, Maimouna Toliver and Fabio Verani contributed to the writing and editing of the manuscript, as well as overall program implementation. All authors mentioned in the article approved the manuscript.

\section{References}

1. Sarkar NN. (2008). The impact of intimate partner violence on women's reproductive health and pregnancy outcome. J Obstet Gynaecol;28(3):26671.

2. Campbell, J.C. (2012) The health consequences of intimate partner violence. Lancet, 359(9314):1131336

3. Garcia-Moreno C., Jansen H.A., Ellsberg M., Heise L., Watts C.H. (2006) Prevalence of intimate partner violence: findings from the WHO multi-country study on women's health and domestic violence. Lancet, 368(9543):1260-9.

4. WHO. (2013). Global and regional estimates of violence against women: prevalence and health effects of intimate partner violence and non-partner sexual violence. Geneva: WHO.

5. Stockl H., Devries K., Rotstein A., Abrahams N., Campbell J., Watts C., et al. (2013) The global prevalence of intimate partner homicide: a systematic review. Lancet, 382(9895):859-65.

6. Stockl H., March L., Pallitto C., Garcia-Moreno C. (2014). Intimate partner violence among adolescents and young women: prevalence and associated factors in nine countries: a crosssectional study. BMC Public Health 14:751.
7. Rahman M., Nakamura K., Seino K., Kizuki M. (2012). Intimate partner violence and use of reproductive health services among married women: evidence from a national Bangladeshi sample. BMC Public Health 12:913.

8. Institut National des Statistiques (2014) Enquete Demographique et de sante - Etude a indicateurs multiples (EDS-MICS), Guinee 2012. Conakry: INS; Macro Int.

9. Ministere des Affaires Sociales G. Enquête nationale sur la violence á l'égard des femmes et des hommes. Conakry: Ministere des Affaires Sociales, UNFPA, UNICEF; 2009.

10. Guedes A., Bott S., Cuca Y. (2002) Integrating systematic screening for gender-based violence into sexual and reproductive health services: results of a baseline study by the International Planned Parenthood Federation, Western Hemisphere Region. Int J Gynaecol Obstet, 78 (1): S57-S63.

11. Rinehart W, Rudy S, Drennan M. GATHER guide to counseling. Popul Rep J. 1998 Dec;(48):1-31.

12. Delamou, A., and Samandari, G. 2014. Integrating intimate partner violence screening and counseling with family planning services: Experience in Conakry, Guinea. The RESPOND Project Study Series: Contributions to Global KnowledgeReport No. 16. New York: Engender Health (The RESPOND Project). Available at http://www. respond-project.org/archive/files/6/6.2/Study16Integrating-IPV-and-FP.pdf

13. Delamou A, Samandari G, Camara BS, Traore P, Diallo FG, Millimono S, Wane D, Toliver M, Laffe K, Verani F. Prevalence and correlates of intimate partner violence among family planning clients in Conakry, Guinea. BMC Res Notes. 2015 Dec 23;8:814. doi: 10.1186/s13104-015-1811-7.

14. Christofedes N, Jewkes R. Acceptability of universal screening for intimate partner violence in voluntary HIV testing and counseling services in South Africa and service implications. AIDS Care. 2010; 22(3): 279-285

15. Bair-Merritt MH, Lewis-O'Connor A, Goel S, Amato P, Ismailji $\mathrm{T}$, Jelley $\mathrm{M}$, Lenahan $\mathrm{P}$, Cronholm $\mathrm{P}$. Primary Care-Based Interventions for Intimate Partner Violence: A Systematic Review. Am J Prev Med. 2014 24(2); 188-194

16. Sabo B. Reflecting on the concept of compassion fatigue. Online J Issues Nurs. 2011;16(1).

17. McCann IL, Pearlman LA. Vicarious traumatization: A framework for understanding the psychological effects of working with victims. J Trauma Stress. 1990;3(1):131-149.

18. Waalen J., Goodwin MM., Spitz AM, Petersen R, Saltzman LE. Screening for intimate partner violence by health care providers: Barriers and interventions. Am J Prev Med. 2000 Nov; 19(4):230-237.

19. Moracco KE, Cole TB. Preventing Intimate Partner Violence: Screening Is Not Enough.JAMA. 2009;302(5):568-570. 